\title{
Lophotrochozoa Get into the Game: The Nodal Pathway and Left/Right Asymmetry in Bilateria
}

\author{
C. GRANDE ${ }^{1}$ AND N.H. PATEL ${ }^{2}$ \\ ${ }^{1}$ Centro de Biología Molecular Severo Ochoa. Universidad Autónoma de Madrid. \\ 28049, Madrid, Spain; ${ }^{2}$ Departments of Molecular and Cell Biology and of Integrative \\ Biology, University of California, Berkeley, California 94720-3200 \\ Correspondence:nipam@uclink.berkeley.edu
}

\begin{abstract}
Animals as diverse as humans, flies, crabs, and snails show overall bilateral symmetry, but each species has specific structures and organs that display left/right asymmetry, and the presence of these asymmetries is vital to the organism. Here, we review recent results showing that part of the molecular pathway that sets left/right asymmetry in vertebrates is also conserved in snails, suggesting that left/right asymmetry was present in the common ancestor of all bilaterians. More specifically, we can now predict that the signaling molecule Nodal and the transcription factor Pitx were expressed on the right side of the bilaterian ancestor. These results also allow us to understand how the direction of shell coiling (chirality) is regulated in snails and provides interesting insights into the possible inversion of the dorsoventral axis in the lineage leading to chordates.
\end{abstract}

Despite great variation in form, most animals are classified as bilaterians because they possess clear anteroposterior (AP) and dorsoventral (DV) axes, which then together position the axis of bilateral symmetry separating the left and right sides of the body. However, although bilaterians show a general symmetry between the left and right sides of the body, most deviate from perfect symmetry in some reproducible way. This can be as subtle as the direction of looping of an internal organ in a fly or as obvious as the asymmetric specialization of claws in certain crab species. Other examples of left/right (LR) asymmetry include the positioning of organs, such as the heart and liver in humans, and the LR asymmetric growth pattern of snail shells that leads to their coiling in either clockwise or anticlockwise directions. Some of these asymmetries are essential to properly pack and connect many internal organs. Individuals that are perfect mirror images of normal are generally viable, whereas individuals in which there are no asymmetries, or the pattern of asymmetry is random between organs, are often severely compromised.

Phylogenetic studies agree on the existence of three main groups within the Bilateria (Aquinaldo et al. 1997): Deuterostomia (including echinoderms, hemichordates, and chordates), Ecdysozoa (including arthropods and nematodes, among others), and Lophotrochozoa (including, for example, snails and annelids); the Ecdysozoa and Lophotrochozoa are closer to each other than either is to Deuterostomia. Although examples of LR asymmetry can be found in representatives of these three main groups, the molecular mechanisms controlling the development of this asymmetry have been studied in detail in just a few model systems in Deuterostomia and Ecdysozoa. The initial identification of several genes that are asymmetrically expressed with respect to the midline in vertebrates (Levin et al. 1995), combined with subsequent functional studies, led to rapid progress in understanding the molecular and genetic mechanisms underlying LR asymmetry in the deuterostomes. These studies led to the identification of Nodal, a transforming growth factor- $\beta$ (TGF- $\beta$ ) ligand, as a signaling molecule that is asymmetrically expressed on the LR axis and functionally critical for establishing LR asymmetry in all deuterostomes studied so far (Hamada et al. 2002; Morokuma et al. 2002; Yu et al. 2002; Duboc and Lepage 2008). Another TGF- $\beta$ family member, Lefty, and the homeobox gene Pitx2, are also key components of the LR pathway (Boorman and Shimeld 2002; Hamada et al. 2002; Duboc et al. 2005).

Like nodal, lefty is also asymmetrically expressed transiently, and both genes positively regulate Pitx, which is critical in cellular processes of differential migration, proliferation, adhesion, and asymmetric morphogenesis in deuterostomes. Loss of expression, as well as inappropriate spatial misexpression of these genes, leads to heterotaxia, i.e., the abnormal LR arrangement of organs (Levin et al. 1997; Sampath et al. 1997). Comparative studies in deuterostomes (echinoderms, ascidians, Amphioxus, and vertebrates) have revealed that nodal, lefty, and Pitx have evolutionarily conserved roles in development and mechanisms of regulation (Hamada et al. 2002; Duboc et al. 2005; Shimeld and Levin 2006).

In contrast, this particular cascade of genes that regulates LR asymmetries in all deuterostomes is not evolutionarily conserved in at least some, if not all, Ecdysozoa. To date, no nodal or lefty orthologs have been identified in the genomes of model ecdysozoans, and although an ortholog of Pitx is present in Drosophila, this gene shows a bilaterally symmetric expression pattern, and no LR defects are seen in Drosophila Pitx mutants (Vorbrüggen et al. 1997), suggesting that some other mechanisms must be involved in the specification of LR asymmetry in Drosophila and possibly other Ecdysozoa. The identification of some 
Drosophila mutants with altered LR asymmetry patterns (fas2, sim, puc, hkb, byn, ptc, and myo31DF among others; for review, see Coutelis et al. 2008; Okumura et al. 2008) has provided some clues about the mechanisms that establish LR asymmetry in this group, but further studies are needed to understand the potential links between LR asymmetry in deuterostomes and ecdysozoans.

Initial data on the phylogenetic distribution of nodal orthologs (present in deuterostomes but absent in ecdysozoans and cnidarians) suggested that the Nodal pathway first originated in the common ancestor of deuterostomes (Fig. 1). However, the recent discovery of a nodal ortholog in snails and annelids (Grande and Patel 2009), both members of the Lophotrochozoa, has established a new hypothesis in which the Nodal pathway evolved before the bilaterians split off into the Ecdysozoa, the Lophotrochozoa, and the Deuterostomia (Fig. 1). Below, we describe the implications of these striking new data on the origin and evolution of the Nodal pathway and LR asymmetry within an evolutionary perspective.

\section{LR ASYMMETRY IN SNAILS}

On the basis of direction of shell coiling and body organization, snails can be left-handed (sinistral, shell coiled in an anticlockwise direction) or right-handed (dextral, shell coiled in a clockwise direction), with dextral coiling as the ancestral configuration as suggested by the fossil record (Ponder and Lindberg 1997). These two mirror-imaged configurations are a manifestation of LR asymmetry in snails. Most snail species are dextral, but a few are sinistral. In a small number of cases, both types can exist within a population of a single species. Classical genetic studies showed that the handedness of snails is genetically determined by a maternal effect locus (Boycott and Diver 1923; Sturtevant 1923; Boycott et al. 1930; Freeman and Lundelius 1982), but the molecular nature of the gene involved remains unknown. The handedness of a snail can be detected during the third cell division of the embryo when the first spiral cleavage occurs (Meshcheryakov and Beloussov 1975). The spiral cleavage is characterized by the oblique angle of the early cleavage planes and the alternating directions of successive divisions. For dextral individuals, the third cleavage generates a set of four micromeres that are oriented with a clockwise spiral relative to their underlying macromere sisters, whereas a sinistral individual displays the opposite rotation pattern.

Recent work on dextral and sinistral forms of the snail Lymnaea stagnalis has shown that cytoskeletal dynamics have a crucial role in determination of body handedness (Shibazaki et al. 2004). In this case, helical spindle inclination and clockwise spiral micromere formation are observed in the dominant dextral embryos at the meta-

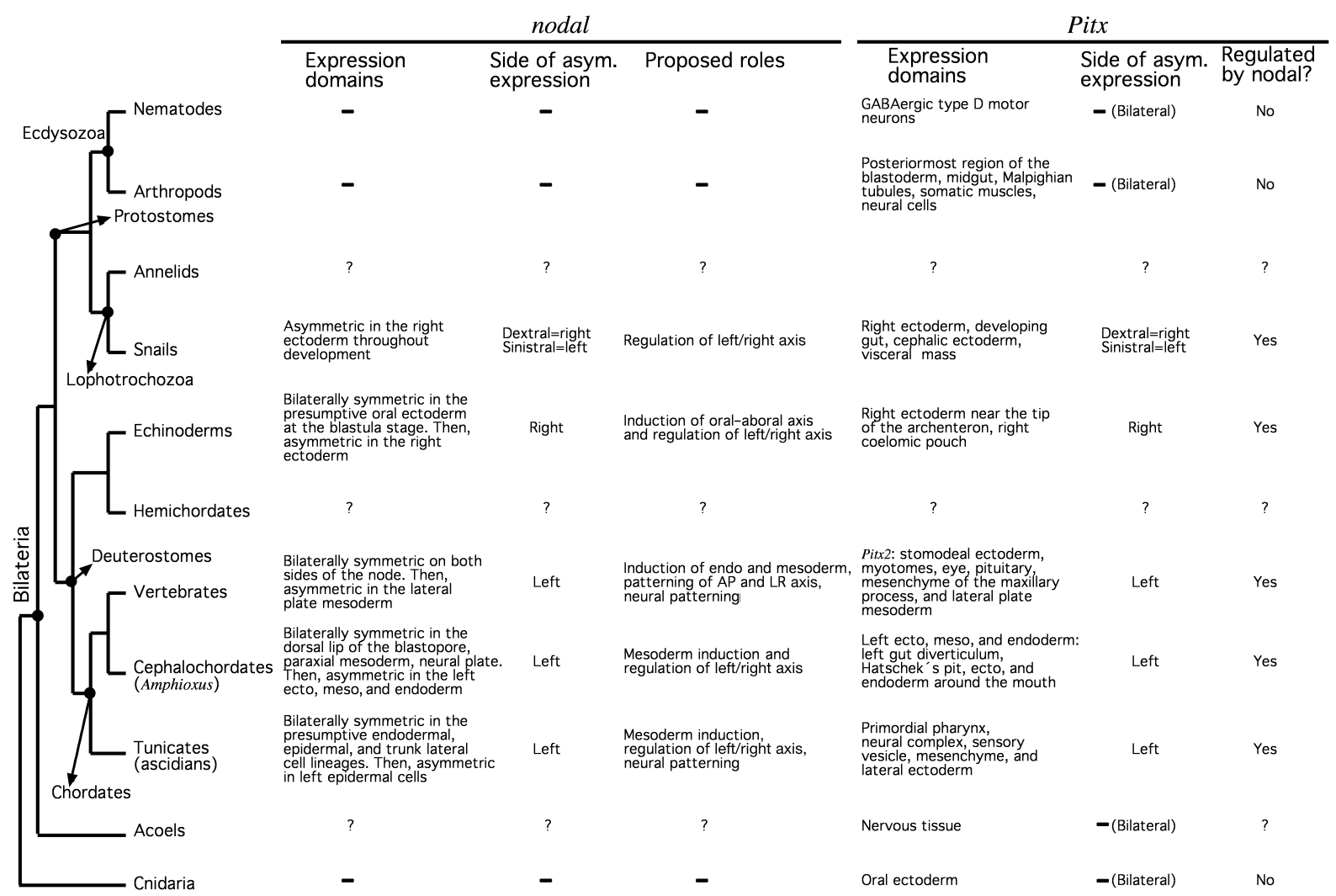

Figure 1. Phylogenetic relationships and nodal and Pitx functions among Bilateria. For each group, expression domains and side of the body that expresses nodal and Pitx are shown. Proposed roles for nodal and the relationship between nodal and Pitx are also given. Question marks indicate that no information is available. Boldface dashes indicate that the gene has not been reported in this group. The same information is also provided for Acoels and Cnidaria. 
phase-anaphase transition initiating the third division, whereas in the recessive sinistral embryos, the third cleavage at first appears to be radially oriented, and helicity first emerges during furrow formation. The actual importance of this cleavage pattern to overall chirality has been tested by mechanically manipulating the orientation of the third cleavage (Kuroda et al. 2009). If an embryo that would have developed as a dextral animal is mechanically manipulated so that the micromeres are forced to rotate in an anticlockwise spiral (opposite to what would have normally occurred), the subsequently formed individual is instead sinistral. Likewise, if an embryo that would have developed as a sinistral animal is mechanically manipulated so that the micromeres are forced to rotate in a clockwise spiral (opposite to what would have normally occurred), the subsequently formed individual is instead dextral.

Interestingly, correct spindle orientation by a $\mathrm{G}$ protein at the third division has been also shown to be critical for LR asymmetry in the nematode Caenorhabditis elegans (Wood 1991; Bergmann et al. 2003). Although G proteins have been also shown to be involved in cytoskeletal spindle alignment in Drosophila (Ahringer 2003), it is unknown if this has any role in LR asymmetry for Drosophila. Nevertheless, actin and associated molecular motors such as myosins have been shown to be involved in LR determination in Drosophila (Speder et al. 2006) and vertebrates (Danilchik et al. 2006). These results support the role of the actin cytoskeleton in defining early LR asymmetry in Bilateria, although further molecular and cellular work are needed to understand the actual mechanisms behind these observations. An interesting hypothesis proposes the cytoskeleton as a vector for orienting key polarized molecules within cells (Aw and Levin 2009), but further studies are needed to test this hypothesis.

A widely accepted mechanism of symmetry breaking in vertebrates involves the rotation of cilia and the generation of a leftward flow in or close to the embryonic node in several vertebrates (Nonaka et al. 2002; Kramer-Zucker et al. 2005; Schweickert et al. 2007). One model is that nodal expression is initially symmetric, but the leftward flow at the node causes a leftward bias in the concentration of Nodal protein, and through its own autoregulation, nodal then becomes asymmetrically expressed on the left side. Supporting this model are the observations that mutations affecting inversin and motor proteins that modify nodal flow cause LR defects in several vertebrates (Afzelius 1976; Supp et al. 1997). Recent studies in zebrafish and frogs have shown a role for fibroblast growth factor signaling in LR regulation through ciliogenesis (Hong and Dawid 2009; Neugebauer et al. 2009). These results support the key role of cilia in LR asymmetry in vertebrates. However, the observation of an early leftward movement of cells around the node in chicks before the appearance of cilia suggests that alternative modes of symmetry breaking are possible within vertebrates (Gros et al. 2009). Furthermore, the fact that modification of ciliogenesis in snails (Shibazaki et al. 2004) and flies (Speder et al. 2006) does not lead to LR defects, together with the fact that asymmetrical nodal expression in snails starts long before the appearance of motile cilia (Grande and Patel 2009; see below), suggests that cilia may not be involved in LR determination in snails and flies.

Ion flow has also been described as a mechanism for early symmetry breaking in deuterostomes (excluding mammals) (Levin 2005) and nematodes (Bauer Huang et al. 2007). The ion flow could actively transport small LR determinants through gap junctions to one side, where they would initiate asymmetric regulation. However, no information regarding the potential role of ion flow in Lophotrochozoa is currently available and thus should be addressed in future studies to explore the possibility that this is an evolutionary ancient mechanism for symmetry breaking.

In summary, although further studies are required, current data support the existence of different asymmetrybreaking mechanisms among the Bilateria. However, the conserved subsequent asymmetric activation of the Nodal pathway in Deuterostomia and Lophotrochozoa (see below) argues that, despite variation in the mechanisms of the initial symmetry-breaking event, the LR asymmetry pathways of bilaterians converge on generating the asymmetric expression of nodal and traces this asymmetric expression of nodal back to the ancestor of all bilaterians.

\section{THE NODAL PATHWAY IN BILATERIA: AN EVOLUTIONARY PERSPECTIVE}

Comparative molecular analysis of nodal in deuterostomes and lophotrochozoans has turned out to be useful in determining the extent to which the LR asymmetry pathway is conserved in evolution and in inferring the ancestral features of the Nodal pathway in Bilateria. As described above, nodal was initially believed to be an innovation of the deuterostomes, but the discovery of a nodal ortholog in Lophotrochozoa (Grande and Patel 2009) suggested that Nodal predated the separation of the three main bilaterian lineages (see Fig. 1). Furthermore, the finding that nodal is asymmetrically expressed along the LR axis and functions in LR asymmetry in snails suggests that the role of the Nodal signaling pathway in LR asymmetry also predates the split of the Bilateria.

Several evolutionary relevant issues can be highlighted here. First, as described previously, nodal expression in vertebrates is initially symmetrical and depends on its own regulation in a positive and negative feedback loop to achieve an asymmetric pattern (Hamada et al. 2002). As in vertebrates, nodal expression is also first symmetric and then later asymmetric in nonvertebrate deuterostomes (Morokuma et al. 2002; Yu et al. 2002; Duboc et al. 2005). But in contrast, nodal expression in snails is asymmetric from the onset (early cleavage stages), and it does not seem to be regulated by its own expression (Grande and Patel 2009). The previously described differences in symmetry-breaking mechanisms observed in different bilaterians might be responsible for these differences in the onset of nodal expression.

Second, nodal expression (as it relates to LR asymmetry) occurs in different germ layers depending on the group considered: the ectoderm in echinoderms, the three germ layers in Amphioxus and probably in ascidians, and the mesoderm in vertebrates (Hamada et al. 2002; Morokuma 
et al. 2002; Yu et al. 2002; Duboc et al. 2005). The restriction of nodal expression exclusively to the ectoderm in snails and echinoderms suggests that the ancestral state is ectodermal and that it was expanded to the three germ layers in the ancestor of chordates and secondarily restricted to the mesoderm in vertebrates (Chea et al. 2005).

Third, the functional significance of the Nodal pathway outside of LR patterning (e.g., in gastrulation) also seems to vary among different bilaterians. The Nodal pathway is essential for endoderm and mesoderm formation in vertebrates (Shen 2007) and mesoderm patterning in Amphioxus (Yu et al. 2002). In ascidians, however, nodal signaling is not critical for endoderm and mesoderm specification because only secondary muscle induction seems to require nodal signaling (Hudson and Yasuo 2005). In addition, echinoderms specify germ layers independently of nodal activity (Duboc and Lepage 2008). On the other hand, modification of the Nodal pathway in snails produces embryos that fail to gastrulate (Grande and Patel 2009), suggesting that germ-layer specification may require the Nodal pathway, although more thorough studies are required to confirm the generality of this finding.

The Nodal pathway also has a role in the regulation of the AP and DV axes in vertebrates (Hamada et al. 2002) as well as the oral-aboral axis in echinoderms (Duboc et al. 2004). Preliminary data on the disruption of the Nodal pathway in snails show modifications in LR morphology, although neither the AP nor DV axis seems to be modified (Grande and Patel 2009). Hence, currently available data support the ancestral function of Nodal signaling in LR asymmetry in Bilateria. Functional manipulation of nodal orthologs in Amphioxus and ascidians, as well as additional lophotrochozoans, will clarify the evolutionary history of the role of nodal in DV and AP axis regulation.

Besides germ-layer induction and axis specification, Nodal signaling is also required in neural patterning in vertebrates (Shen 2007) and ascidians (Hudson and Yasuo 2005). In vertebrates, generation of anterior neuronal tissue requires inhibition of signaling, although high levels of nodal activity in the posterior epiblast are necessary for ventral patterning of the neural tubes and maintenance of anterior forebrain territories (Shen 2007). In contrast, Nodal signaling is required for lateral fate in the neural tube of ascidians (Hudson and Yasuo 2005). In snails, nodal transcription is seen in the cephalic regions but in an asymmetric pattern. Whether this expression correlates to LR symmetries in the nervous system in snails is still unknown. If true, however, this would support the hypothesis that nodal has an ancestral role in regulating brain asymmetry (Palmer 2004).

The Nodal signaling pathway acts on the left side in vertebrates, and it is a determinant for leftness in this group because inappropriate induction of nodal activity on the right side produces left-side morphologies (Hamada et al. 2002). All other deuterostomes conserve this pattern of expression except for echinoderms, which show Nodal signaling on the right side (Duboc et al. 2005). Unlike vertebrates, Nodal signaling is a determinant for rightness in echinoderms because inappropriate induction of nodal activity on the left side produces right- side morphologies and prevents the formation of the rudiment (an imaginal structure that gives rise to most of the adult organs and is normally present on just the left side). This suggests that the rudiment normally forms on the left side by default (Duboc et al. 2005). Thus, in echinoderms, the presence of nodal on the right modifies the "default" condition. In snails, the side that expresses nodal is correlated with chirality (i.e., the right side in dextral snails and the left in sinistral snails) (Grande and Patel 2009). The first indication of morphological asymmetry in snails is given by a displacement of the shell gland to the left in dextral species and to the right in sinistral species due to a differential formation of shell-producing cells on one side of the embryo. Inhibition of the Nodal pathway in sinistral snails produces noncoiled shells (Grande and Patel 2009), which suggests equal formation of shell-producing cells on both sides of the embryo or, in other words, that the default condition is established on both sides of the embryo when nodal function is eliminated. In summary, these results suggest that the side of the embryo that expresses nodal is the one that acquires new features and defers from the default condition.

Finally, several lines of evidence indicate that the last common ancestor of snails had a dextral body (Ponder and Lindberg 1997) and therefore expressed nodal on the right side. This, together with the fact that nodal is also expressed on the right side in echinoderms, raises the hypothesis that the bilaterian ancestor expressed nodal on the right side as well.

\section{CONSERVED GENE CASCADE NODAL-PITX IN BILATERIA}

Pitx is a bicoid-type homeobox transcription factor that shows multiple sites of expression during deuterostome development. Two roles have been described for Pitx genes in chordates: regulation of the pituitary and its homologs and control of directional asymmetry in visceral organ organization (Boorman and Shimeld 2002). Data from echinoderms, ascidians, Amphioxus, and vertebrates show that this gene is important in asymmetric morphogenesis and that it is up-regulated by nodal (Boorman and Shimeld 2002; Hamada et al. 2002; Yoshida and Saiga 2008). Following the asymmetric expression of nodal, Pitx is also asymmetrically expressed, persists longer than nodal, and is localized in the primordia of most asymmetric organs. Although Pitx orthologs have been described for members of the Ecdysozoa (Drosophila and C. elegans) (McIntire et al. 1993; Vorbrüggen et al. 1997), asymmetrical expression patterns have not been reported in this group, and functional experiments suggest that Pitx is not involved in pattern formation during development in Ecdysozoa. Interestingly, a Pitx ortholog has been recently identified in snails, and, like deuterostomes and unlike ecdysozoans, Pitx is asymmetrically expressed and regulated by nodal (Grande and Patel 2009). Asymmetric expression of Pitx in snails starts right after the activation of nodal and also correlates with body handedness (it is expressed on the right side in dextral snails and on the left in sinistral snails) (Grande and Patel 2009). Hence, these new data suggest that the ancestor of bilateri- 
ans already had the gene cascade nodal-Pitx controlling LR asymmetry (see Fig. 1).

In addition to asymmetric expression, Pitx is a stomodeal (mouth) marker for both chordates and echinoderms (Christiaen et al. 2007). However, in another group of deuterostomes, hemichordates, this gene is expressed at the opposite end of the site of mouth formation (Lowe et al. 2006). This result, together with the absence of stomodeal expression of the Pitx ortholog in ecdysozoans (McIntire et al. 1993; Vorbrüggen et al. 1997), initially argued against stomadeal expression as an ancestral state in deuterostomes. However, new data from snails show that Pitx is also expressed in the stomodeum (Grande and Patel 2009) and therefore supports the hypothesis that Pitx was expressed not only in the stomodeum of the ancestor of deuterostomes, but also in the stomodeum of the ancestor of bilaterians.

Finally, the Pitx transcript in snails is localized simultaneously in different asymmetric and symmetric domains (Grande and Patel 2009). Preliminary data on the inhibition of the Nodal pathway in snails show that nodal regulates exclusively the asymmetric domain of Pitx expression, suggesting different independent enhancers for Pitx in snails, as described for vertebrates (Christiaen et al. 2005, 2007).

\section{THE DV INVERSION HYPOTHESIS AND THE LR AXIS}

In the 19th century, Geoffroy Saint-Hilaire (1822) proposed that the DV axis of deuterostomes is inverted with respect to that observed in protostomes (Lophotrochozoa plus Ecdysozoa). He based this idea on the relative placement of the nerve cord (ventral in protostomes and dorsal in deuterostomes) and the heart and circulatory vessels (dorsal in protostomes and ventral in deuterostomes). Although this hypothesis was eventually dismissed, recent molecular data on developmental expression and function of homologous genes in distantly related taxa have been interpreted as new evidence for the inversion of the axis (Arendt and NüblerJung 1994; Holley et al. 1995; DeRobertis and Sasai 1996; Gerhart 2000). Several orthologous genes of chordates and protostomes show similar but inverted expression domains. This is especially striking in those related with DV patterning, such as certain TGF- $\beta$ ligands (Bmp4 in chordates and its ortholog Dpp in Drosophila) and TGF- $\beta$ inhibitors (Chordin in chordates and its ortholog Sog in Drosophila). bmp4 is expressed in ventral domains and has ventralizing activity in chordates, whereas chordin is expressed dorsally and has an antagonistic role (Holley et al. 1995; DeRobertis and Sasai 1996). On the other hand, $d p p$ is expressed in dorsal domains and has dorsalizing activity in Drosophila, whereas $\operatorname{sog}$ is expressed in ventral domains and has an antagonistic role (Holley et al. 1995; DeRobertis and Sasai 1996). However, both chordin and sog promote the development of the central nervous system wherever they are expressed, suggesting that the ventral nerve cord of insects and the dorsal nerve cord of chordates are built by the same genetic machinery and may have not evolved independently. In addition - hedgehog, an important gene in dorsal midline formation, differentiation of neural-tube structures, and DV patterning of adjacent tissues in chordates (Sasai and DeRobertis 1997) - is expressed in the ventral midline in Lophotrochozoa (Nederbragt et al. 2002), suggesting that similar mechanisms are involved in the development of the midline in both groups and adding new evidence for DV axis inversion. Furthermore, several other genes involved in heart and nervous system development, such as $N k \times 2-5$, netrin, vnd/Nk2, ind/Gsh1,2 and Msh/Msx1,3, are expressed in opposite domains in chordates and protostomes (Weiss et al. 1998; Tanaka et al. 1999). Thus, on the basis of both morphological and molecular data, the DV inversion hypothesis postulates that the common ancestor of deuterostomes and protostomes already had a nerve cord, a heart, and circulatory vessels, as well as the genetic modules to build them, and that the DV axis was inverted throughout the course of evolution in one of the two lineages.

The LR axis is always specified with reference to AP and DV axes. Experimental manipulations have shown that the LR axis can be reversed by reversing the AP axis or the DV axis (Ligoxygakis et al. 2001). Thus, according to the DV inversion hypothesis, genes that are expressed on one side of the body (left versus right) in one of the two groups (either protostomes or deuterostomes) are expected to be expressed on the opposite side in the other group. This is exactly the case for the nodal-Pitx gene cascade, which is expressed on the right side in dextral snails (representing the ancestral state) (Grande and Patel 2009) and on the left side in chordates (Hamada et al. 2002). The fact that nodalPitx is also expressed on the right side in echinoderms (Duboc et al. 2005) suggests that the inversion must have occurred in the chordate lineage.

Some alternatives to the DV inversion hypothesis have been proposed. Some investigators have suggested that the differences in morphogenetic movements during gastrulation of protostomes versus deuterostomes result in inverted expression patterns of developmental genes (van den Biggelaar et al. 2002). In chordates, the AP axis is extended evenly during gastrulation, whereas in protostomes, the growth along the AP axis is uneven, almost exclusively limited to the dorsal side. Thus, originally, dorsal cells will be located in a ventral position in protostomes after gastrulation, explaining the ventral expression patterns of certain genes with respect to chordates (van den Biggelaar et al. 2002). However, these differences in migration of cells and growth along the AP axis would not explain left versus right differences in expression patterns of the nodal-Pitx cascade described above. In addition, it is important to note that nodal initiates its expression before gastrulation and hence before those morphogenetic movements and uneven growth. Furthermore, the onset of the expression of the ortholog of $d p p$ in snails is also detected in the dorsal domain of the late blastula (C Grande and NH Patel, unpubl.), whereas it is ventral in the early gastrula of vertebrates. Hence, the shift of initially dorsally specified cells to a secondarily ventral position does not fully explain the inverted gene expression domains described above.

Other investigators have suggested that the DV axis of the last common ancestor of protostomes and deuterostomes was relatively undifferentiated, and subsequently both lineages organized the nerve cord and organogenesis independ- 
ently in opposite locations, and thus inversion never occurred (Gerhart 2000). This hypothesis was traditionally based on the presence of a diffuse nerve net in some hemichordates, which supports an undefined central nervous system for the ancestor of deuterostomes. However, recent studies have shown that the nervous system of hemichordates is much more localized than previously thought and therefore that the centralization of the deuterostome nervous system predates the origin of chordates (Nomaksteinsky et al. 2009). Whether nervous system centralization does or does not predate the spilt between deuterostomes and protostomes, the observations on nodal expression are still most consistent with a scenario of some type of DV axis inversion. Further studies of cell and morphological homologies, as well as the gene modules that regulate body organization in a comparative phylogenetic framework, will help to elucidate the evolution of the DV axis and hence of the LR axis as well.

\section{CONCLUSIONS}

A central focus in evolutionary developmental biology is to understand the evolution of the mechanisms that establish and elaborate the body axes of the developing embryo. Recent studies have suggested that DV axis specification is controlled by ancient molecular patterning mechanisms, but whether conserved mechanisms regulate LR asymmetries constitutes an open question for which initial answers have been only recently proposed. Striking support for an ancient origin of LR axis patterning is provided by data on the Nodal pathway in Lophotrochozoa and its function in LR regulation. These results provide evidence of the ancient role of this pathway in LR patterning in Bilateria and sheds light on the evolutionary events that have led to the current diversity in body plans. Finally, new avenues of research are opening up novel approaches to understanding symmetry-breaking events in bilaterians, the regulatory mechanisms involved in LR determination, and the evolutionary implications of variation in these pathways for generating morphological diversity.

\section{ACKNOWLEDGMENTS}

C.G. is currently a "Ramon y Cajal" postdoctoral fellow supported by the Spanish Ministerio de Ciencia e Innovacion and the Universidad Autonoma de Madrid.

\section{REFERENCES}

Afzelius BA. 1976. Human syndrome caused by immotile cilia. Science 193: 317-319.

Aguinaldo AM, Turbeville JM, Linford LS, Rivera MC, Garey JR, Raff RA, Lake JA. 1997. Evidence for a clade of nematodes, arthropods and other moulting animals. Nature 387: 489-493.

Ahringer J. 2003. Control of cell polarity and mitotic spindle positioning in animal cells. Curr Opin Cell Biol 15: 73-81.

Arendt D, Nübler-Jung K. 1994. Inversion of dorsoventral axis? Nature 371: 26.

Aw S, Levin M. 2009. Is left-right asymmetry a form of planar cell polarity? Development 136: 355-366.

Bauer Huang SL, Saheki Y, VanHoven MK, Torayama I, Ishihara T, Katsura I, van der Linden A, Sengupta P, Bargmann CI. 2007.
Left-right olfactory asymmetry results from antagonistic functions of voltage-activated calcium channels and the Raw repeat protein OLRN-1 in C. elegans. Neural Dev 2: 24.

Bergmann DC, Lee M, Robertson B, Tsou MF, Rose LS, Wood WB. 2003. Embryonic handedness choice in C. elegans involves the G $\alpha$ protein GPA-16. Development 130: 5731-5740.

Boorman CJ, Shimeld SM. 2002. Pitx homeobox genes in Ciona and Amphioxus show left-right asymmetry is a conserved chordate character and define the ascidian adenohypophysis. Evol Dev 4: 354-365.

Boycott AE, Diver C. 1923. On the inheritance of sinistrality in Limnaea peregra. Proc R Soc Lond B Biol Sci 95: 207-213.

Boycott AE, Diver C, Garstang SL, Hardy MAC, Turner FM. 1930. The inheritance of sinistrality in Limnaea peregra. Philos Trans R Soc Lond B Biol Sci 219: 51-130.

Chea HK, Wright CV, Swalla BJ. 2005. Nodal signaling and the evolution of deuterostome gastrulation. Dev Dyn 234: 269-278.

Christiaen L, Bourrat F, Joly JS. 2005. A modular cis-regulatory system controls isoform-specific pitx expression in ascidian stomodaeum. Dev Biol 277: 557-566.

Christiaen L, Jaszczyszyn Y, Kerfant M, Kano S, Thermes V, Joly JS. 2007. Evolutionary modification of mouth position in deuterostomes. Semin Cell Dev Biol 18: 502-511.

Coutelis JB, Petzoldt AG, Speder P, Suzanne M, Noselli S. 2008. Left-right asymmetry in Drosophila. Semin Cell Dev Biol 19: 252-262.

Danilchik MV, Brown EE, Riegert K. 2006. Intrinsic chiral properties of the Xenopus egg cortex: An early indicator of left-right asymmetry? Development 133: 4517-4526.

DeRobertis EM, Sasai Y. 1996. A common plan for dorsoventral patterning in Bilateria. Nature 380: 37-40.

Duboc V, Lepage T. 2008. A conserved role for the nodal signaling pathway in the establishment of dorso-ventral and left-right axes in deuterostomes. J Exp Zool B Mol Dev Evol 310: 41-53.

Duboc V, Rottinger E, Besnardeau L, Lepage T. 2004. Nodal and $B M P 2 / 4$ signaling organizes the oral-aboral axis of the sea urchin embryo. Dev Cell 6: 397-410.

Duboc V, Rottinger E, Lapraz F, Besnardeau L, Lepage T. 2005. Left-right asymmetry in the sea urchin embryo is regulated by nodal signaling on the right side. Dev Cell 9: 147-158.

Freeman G, Lundelius J. 1982. The developmental genetics of dextrality and sinistrality in the gastropod Lymnaea peregra. Wilhelm Roux's Arch Dev Biol 191: 69-83.

Geoffroy St. Hillaire E. 1822. Considerations generales sur la vertebre. Mem Mus Hist Nat 9: 89-119.

Gerhart J. 2000. Inversion of the chordate body axis: Are there alternatives? Proc Natl Acad Sci 97: 4445-4448.

Grande C, Patel NH. 2009. Nodal signalling is involved in leftright asymmetry in snails. Nature 457: 1007-1011.

Gros J, Feistel K, Viebahn C, Blum M, Tabin C. 2009. Cell movements at Hensen's node establish left/right asymmetric gene expression in the chick. Science 324: 941-944.

Hamada H, Meno C, Watanabe D, Saijoh Y. 2002. Establishment of vertebrate left-right asymmetry. Nat Rev Genet 2: 103-113.

Holley SA, Jackson PD, Sasai Y, Lu B, DeRobertis EM, Hoffmann FM, Ferguson EL. 1995. A conserved system for dorsal-ventral patterning in insects and vertebrates involving sog and chordin. Nature 376: 249-253.

Hong SK, Dawid IB. 2009. FGF-dependent left-right asymmetry patterning in zebrafish is mediated by Ier2 and Fibp1. Proc Natl Acad Sci 106: 2230-2235.

Hudson C, Yasuo H. 2005. Patterning across the ascidian neural plate by lateral Nodal signalling sources. Development 132: 1199-1210.

Kramer-Zucker AG, Olale F, Haycraft CJ, Yoder BK, Schier AF, Drummond IA. 2005. Cilia-driven fluid flow in the zebrafish pronephros, brain and Kupffer's vesicle is required for normal organogenesis. Development 132: 1907-1921.

Kuroda R, Endo B, Masanri A, Shimuzu M. 2009. Chiral blastomere arrangement dictates zygotic left-right asymmetry pathway in snails. Nature 462: 790-794.

Levin M. 2005. Left-right asymmetry in embryonic development: A comprehensive review. Mech Dev 122: 3-25. 
Levin M, Johnson RL, Stern CD, Kuehn M, Tabin C. 1995. A molecular pathway determining left-right asymmetry in chick embryogenesis. Cell 82: 803-814.

Levin M, Pagan S, Roberts DJ, Cooke J, Kuehn MR, Tabin CJ. 1997. Left/right patterning signals and the independent regulation of different aspects of situs in the chick embryo. Dev Biol 189: $57-67$.

Ligoxygakis P, Strigini M, Averof M. 2001. Specification of leftright asymmetry in the embryonic gut of Drosophila. Development 128: 1171-1174.

Lowe CJ, Terasaki M, Wu M, Freeman RM, Runft L, Kwan K, Haigo S, Aronowicz J, Lander E, Gruber C, et al. 2006. Dorsoventral patterning in hemichordates: Insights into early chordate evolution. PLoS Biol 4: 1603-1619.

McIntire SL, Jorgensen E, Horvitz HR. 1993. Genes required for Gaba function in Caenorhabditis elegans. Nature 364: 334 337.

Meshcheryakov VN, Beloussov LV. 1975. Asymmetrical rotations of blastomeres in early cleavage of gastropoda. Wilhelm Roux's Arch Dev Biol 177: 193-203.

Morokuma J, Ueno M, Kawanishi H, Saiga H, Nishida H. 2002. HrNodal, the ascidian nodal-related gene, is expressed in the left side of the epidermis, and lies upstream of HrPitx. Dev Genes Evol 212: 439-446.

Nederbragt AJ, van Loon AE, Dictus WJ. 2002. Evolutionary biology: Hedgehog crosses the snail's midline. Nature 417: 811812

Neugebauer JM, Amack JD, Peterson AG, Bisgrove BW, Yost HJ. 2009. FGF signalling during embryo development regulates cilia length in diverse epithelia. Nature 458: 651-654.

Nomaksteinsky M, Rottinger E, Dufour HD, Chettouh Z, Lowe CJ, Martindale MQ, Brunet JF. 2009. Centralization of the deuterostome nervous system predates chordates. Curr Biol 19: $1264-1269$.

Nonaka S, Shiratori H, Saijoh Y, Hamada H. 2002. Determination of left-right patterning of the mouse embryo by artificial nodal flow. Nature 418: 96-99.

Okumura T, Utsuno H, Kuroda J, Gittenberger E, Asami T, Matsuno K. 2008. The development and evolution of left-right asymmetry in invertebrates: Lessons from Drosophila and snails. Dev Dyn 237: 3497-3515.

Palmer AR. 2004. Symmetry breaking and the evolution of development. Science 306: 828-833.

Ponder WF, Lindberg DR. 1997. Towards a phylogeny of gastropod molluscs: An analysis using morphological characters. Zool J Linn Soc 119: 83-265.

Sampath K, Cheng AM, Frisch A, Wright CV. 1997. Functional differences among Xenopus nodal-related genes in left-right axis determination. Development 124: 3293-3302.
Sasai Y, DeRobertis EM. 1997. Ectodermal patterning in vertebrate embryos. Dev Biol 182: 5-20.

Schweickert A, Weber T, Beyer T, Vick P, Bogusch S, Feistel K, Blum M. 2007. Cilia-driven leftward flow determines laterality in Xenopus. Curr Biol 17: 60-66.

Shen M. 2007. Nodal signaling: Developmental roles and regulation. Development 134: 1023-1034.

Shibazaki Y, Shimizu M, Kuroda R. 2004. Body handedness is directed by genetically determined cytoskeletal dynamics in the early embryo. Curr Biol 14: 1462-1467.

Shimeld SM, Levin M. 2006. Evidence for the regulation of leftright asymmetry in Ciona intestinalis by ion flux. Dev Dyn 235: $1543-1553$.

Speder P, Adam G, Noselli S. 2006. Type ID unconventional myosin controls left-right asymmetry in Drosophila. Nature 440: 803-807.

Sturtevant AH. 1923. Inheritance of direction of coilling in Lymnaea. Science 58: 269-207.

Supp DM, Witte DP, Potter SS, Brueckner M. 1997. Mutation of an axonemal dynein affects left-right asymmetry in inversus viscerum mice. Nature 389: 963-966.

Tanaka M, Wechsler SB, Lee IW, Yamasaki N, Lawitts JA, Izumo S. 1999. Complex modular cis-acting elements regulate expression of the cardiac specifying homeobox gene Csx/Nkx2.5. Development 126: 1439-1450.

van den Biggelaar JAM, Edsinger-Gonzales E, Schram FR. 2002. The improbability of dorso-ventral axis inversion during animal evolution, as presumed by Geoffroy Saint Hilaire. Contrib Zool 71: 29-36.

Vorbrüggen G, Constien R, Zilian O, Wimmer EA, Dowe G, Taubert H, Noll M, Jäckle H. 1997. Embryonic expression and characterization of a Ptxl homolog in Drosophila. Mech Dev 68: $139-147$.

Weiss JB, Von Ohlen T, Mellerick DM, Dressler G, Doe CQ, Scott MP. 1998. Dorsoventral patterning in the Drosophila central nervous system: The intermediate neuroblasts defective homeobox gene specifies intermediate column identity. Genes Dev 12: 3591-3602.

Wood WB. 1991. Evidence from reversal of handedness in C. elegans embryos for early cell-interactions determining cell fates. Nature 349: 536-538.

Yoshida K, Saiga H. 2008. Left-right asymmetric expression of Pitx is regulated by the asymmetric Nodal signaling through an intronic enhancer in Ciona intestinalis. Dev Genes Evol 218: 353-360.

Yu JK, Holland LZ, Holland ND. 2002. An amphioxus nodal gene (AmphiNodal) with early symmetrical expression in the organizer and mesoderm and later asymmetrical expression associated with left-right axis formation. Evol Dev 4: 418-425. 


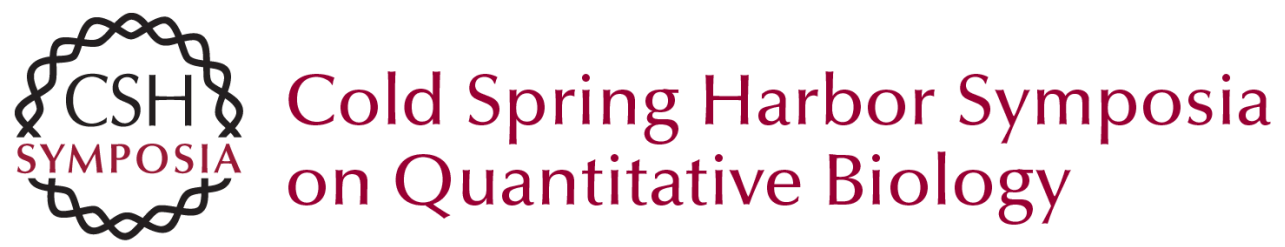

\section{Lophotrochozoa Get into the Game: The Nodal Pathway and Left/Right Asymmetry in Bilateria}

C. Grande and N.H. Patel

Cold Spring Harb Symp Quant Biol 2009 74: 281-287 originally published online April 22, 2010

Access the most recent version at doi:10.1101/sqb.2009.74.044

References This article cites 61 articles, 16 of which can be accessed free at:

http://symposium.cshlp.org/content/74/281.full.html\#ref-list-1

\section{License}

Email Alerting Receive free email alerts when new articles cite this article - sign up in the box at the Service top right corner of the article or click here. 